\title{
Sprachliche Diversität in der Schulsozialarbeit: \\ „Die größte Herausforderung“
}

Marianne Franz (Innsbruck)

\begin{abstract}
School social workers deal with a highly sociolinguistically diverse target group in a monolingually constructed school environment. On the basis of qualitative interviews analysed by means of content analysis, this article traces how school social workers perceive multilingualism in their field of work. Which communicative practices do they use to enable communication in this linguistically diverse environment? Where do they experience challenges? Multilingualism is understood in terms of the language repertoire and includes not only different languages but also different varieties within a language. The school social workers describe a variety of communicative practices ranging from a careful, empathetic linguistic recipient design to multimodal and multimedia practices. Language barriers due to the lack of a shared language, are perceived as the "greatest challenge" that can only be mitigated but not overcome by interpreting services.
\end{abstract}

\section{Soziale und sprachliche Diversität als Kennzeichen und Herausforderung des Berufsfelds Schulsozialarbeit}

Im Fokus steht die in der Linguistik noch wenig beforschte Berufsgruppe der SchulsozialarbeiterInnen. Modelle und Trägerschaft der Schulsozialarbeit sind im deutschsprachigen Raum und oft auch innerhalb der einzelnen Staaten sehr unterschiedlich (cf. Speck 2014: 95-111). Es eint sie das sozialstaatliche Ziel, mittels Einzelberatungen und präventiven oder intervenierenden Unterrichtseinheiten soziale Gerechtigkeit in Form von Bildungs- und Teilhabegerechtigkeit herstellen zu wollen (cf. Emanuel 2017: 16). Zielgruppe der Schulsozialarbeit sind SchülerInnen, Erziehungsberechtigte und LehrerInnen (cf. Speck 2014: 64-66). Der vorliegende Beitrag nimmt exemplarisch Schulsozialarbeit in Österreich in den Blick. Dem Bundesministerium für Bildung, Wissenschaft und Forschung zufolge soll dieses psychosoziale, von der Schulhierarchie unabhängig agierende Unterstützungssystem vor allen Dingen Präventionsarbeit hinsichtlich verschiedenster Themen - von Mobbing, Gewalt, familiären Problemen, Schulklima bis hin zu Liebeskummer - leisten und den Bildungserfolg der einzelnen SchülerInnen fördern (cf. Schulpsychologie/BMBWF). 
Linguistisch ist dieser Beruf aus mehreren Gründen von Interesse: Zentrales Werkzeug der SchulsozialarbeiterInnen ist die Sprache. ${ }^{1}$ Die interaktionalen Anforderungen sind hoch - nicht nur aufgrund der vielfältigen und oft heiklen Themen, sondern auch aufgrund der sprachlich und sozial höchst diversen Zielgruppe, die es zu begleiten und zu beraten gilt. Der Sozialraum Schule gilt unter dem Aspekt der sozialen Diversität als Abbild der Gesellschaft. Die Diversität der InteraktionspartnerInnen ließe sich mit vielerlei soziolinguistischen Kriterien beschreiben: Rolle, Status, Hierarchie, regionale oder nationale Herkunft, Alter, Geschlecht, Religion, Kultur, Identität, Erst- und Zweitsprachen. In diesem Beitrag liegt der Fokus auf der sprachlichen Diversität: So belief sich in den österreichischen Schulen im Schuljahr 2017/2018 der Anteil der SchülerInnen mit einer anderen Umgangssprache als Deutsch, gemeint ist hier die im Alltag hauptsächlich verwendete Sprache, österreichweit auf 26\% - mit großen regionalen Unterschieden (in Wien etwa knapp 52\%) (cf. Expertenrat für Integration 2019: 34). Auch wenn diese Zahl nichts über die tatsächlichen Deutschkompetenzen der SchülerInnen aussagt ${ }^{2}$, gibt sie doch einen Einblick in die mehrsprachige Schulrealität, der der immer noch monolinguale Habitus des österreichischen Bildungssystems nicht gerecht wird (cf. Gogolin 2008; Fleck 2013). SchulsozialarbeiterInnen bewegen sich in diesem monolingual konstruierten, aber faktisch mehrsprachigen Sozialraum Schule, kommen in ihrer Arbeit mit unterschiedlichsten Sprachen und Sprachkompetenzen in Berührung und stoßen mitunter an die Grenzen ihrer Handlungsfähigkeit. Das Fehlen einer gemeinsamen Sprache, aber auch unterschiedliche Sprachkompetenzen der GesprächsteilnehmerInnen (Erstsprache/Zweitsprache) werden von den SchulsozialarbeiterInnen zum Teil als gesprächshindernde Sprachbarriere empfunden. Dabei ist der mehrsprachige Sozialraum Schule deklariertes Aufgabenfeld der Schulsozialarbeit: In Österreich etwa wurde letztere teilweise - nicht unumstritten - explizit an Schulen eingerichtet, die sich durch einen hohen Anteil an SchülerInnen mit anderen Erstsprachen als Deutsch kennzeichnen (in Zusammenhang mit einem hohen Anteil an Eltern mit maximal Pflichtschulabschluss). ${ }^{3}$ Die Installation von Schulsozialarbeit wird politisch auch gerne mit den Anforderungen einer Migrationsgesellschaft und mit ,wachsende[n] Sozialisations- und Integrationsprobleme[n] Jugendlicher und damit einhergehende[n] Herausforderungen für das Schulsystem“ (Schörner/Würfl 2013: 1) begründet.

\footnotetext{
${ }^{1}$ Linguistisch bereits besser beforschte, im Sozialbereich tätige vergleichbare Berufsgruppen wären etwa PsychotherapeutInnen, TelefonseelsorgerInnen, SupervisorInnen, LehrerInnen, ÄrztInnen u.v.m. (cf. hierzu die Beiträge im Sammelband „Beraten in Interaktion“ von Pick 2017) und auch SozialarbeiterInnen, die außerhalb der Schule agieren (cf. Hitzler 2012).

2 Die sogenannte Umgangssprache (im Sinne der am häufigsten im Alltag verwendeten Sprache) gilt in Österreich als integrationsspolitischer Indikator. Klar ist, dass die hier von den SchülerInnen angeführte Sprache nichts darüber aussagt, ob die Person über Deutschkompetenzen verfügt (und auf welchem Niveau) oder nicht. Der Verband für Angewandte Linguistik hat zu einigen Punkten des hier zitierten Integrationsberichtes 2019 ausführlich Stellung genommen (cf. verbal 2019). Bei aller berechtigten Kritik sei diese Statistik an dieser Stelle mangels Alternativen ein Hinweis auf die sprachliche Diversität in den österreichischen Schulen.

${ }^{3}$ Cf. die Finanzierung von Schulsozialarbeit und deren Verteilung auf österreichische Schulen durch den sogenannten Integrationstopf im Zuge der globalen Flüchtlingsbewegungen ab dem Jahr 2016 per „Chancenindex“ (cf. BMB 2016).
} 
Wie im Beitrag noch gezeigt werden wird, gibt es in der Praxis allerdings nur beschränkte sogenannte Top-down-Angebote, die die SchulsozialarbeiterInnen in dieser sprachlich diversen Situation unterstützen sollen (siehe Kapitel 4). So sind die SchulsozialarbeiterInnen gefordert, dementsprechende Bottom-up-Praktiken zu entwickeln, die das Gelingen der Kommunikation ermöglichen. Der Beitrag widmet sich ebendiesen kommunikativen Praktiken und Routinen, die den SchulsozialarbeiterInnen in ihrer Interaktion mit multilingualen GesprächspartnerInnen bewusst sind. Unter einer kommunikativen Praktik wird hier - dem konversationsanalytischen Zugang folgend - der routinisierte ,kontextsensitiv[e] Einsatz von bestimmten sprachlich-kommunikativen Formen als Ressourcen zur Lösung grundlegender Aufgaben der Interaktionskonstitution“"verstanden (Deppermann/Feilke/Linke 2016: 1). Hinsichtlich der Multilingualität wird vom Sprachrepertoire-Begriff (cf. Gumperz 1964; Busch 2017) ausgegangen, der sowohl innere als auch äußere Mehrsprachigkeit umfasst, d. h. neben Fremdsprachenkenntnissen auch innersprachliche Varietäten von Dialekt und Umgangssprache über Soziolekte (Bsp. Jugendsprache) bis hin zur Standardsprache berücksichtigt.

Die Forschungsfragen, die beantwortet werden sollen, lauten:

1. Welche sprachlichen Kompetenzen werden im Umgang mit Mehrsprachigkeit im beruflichen Alltag von den SchulsozialarbeiterInnen relevant gesetzt (z. B. das eigene Sprachrepertoire (cf. Busch 2017; Gumperz 1964)) (Kapitel 3)?

2. Welche Top-down- und Bottom-up-Kommunikationspraktiken im Umgang mit sprachlicher Diversität werden beschrieben (Kapitel 4)? Unter Top-down-Praktiken verstehe ich hier Praktiken, die von den beteiligten Institutionen geschaffen bzw. angeboten werden, um die SchulsozialarbeiterInnen in ihrer Tätigkeit zu unterstützen. Bottom-upPraktiken hingegen werden von den SchulsozialarbeiterInnen und/oder anderen GesprächsteilnehmerInnen selbst kreiert/improvisiert (cf. auch Wilde et al. 2018: 378).

3. Wo situieren die SchulsozialarbeiterInnen Herausforderungen in Zusammenhang mit Mehrsprachigkeit an ihrem Arbeitsplatz (Kapitel 5)?

\section{Daten und Methoden}

Datengrundlage der qualitativen Studie sind ein Experteninterview (cf. Blöbaum/Nölleke/Scheu 2016) sowie eine offene Gruppendiskussion (cf. Lüthje 2016), die teil-strukturiert und teil-narrativ angelegt sind (cf. Deppermann 2013), beide von mir geführt wurden und als Audio- bzw. Audio- und Videoaufnahme vorliegen (Tabelle 1). Alle ProbandInnen arbeiten für die Schulsozialarbeit Tirol, deren Trägerin die Kinder- und Jugend GmbH ist. Die SchulsozialarbeiterInnen verfügen in den von ihnen betreuten Schulen über eigene Räumlichkeiten und sind dort täglich vor Ort. Diese Vor-Ort-Präsenz bei gleichzeitiger struktureller Unabhängigkeit gegenüber der Schulleitung ist Kernkonzept der Schulsozialarbeit und sichert Niederschwelligkeit und Vertraulichkeit des Angebots. 


\begin{tabular}{|c|c|c|}
\hline & 1x Experteninterview & 1x offene Gruppendiskussion \\
\hline TeilnehmerInnen & Leiter der Schulsozialarbeit Tirol & $\begin{array}{l}5 \text { SchulsozialarbeiterInnen, } 1 \\
\text { Person davon nun in administrati- } \\
\text { ver Funktion }\end{array}$ \\
\hline Geschlecht & $\mathrm{m}$ & $1 \mathrm{~m}, 4 \mathrm{w}$ \\
\hline Alter & k. A. & 29-40 Jahre \\
\hline Bildungsabschluss & Magister (FH) Soziale Arbeit & $\begin{array}{l}\text { Bachelor oder Magister (FH) } \\
\text { Soziale Arbeit, Soziale Akademie } \\
\text { (Diplom) }\end{array}$ \\
\hline $\begin{array}{l}\text { Schule der Tätigkeit/ } \\
\text { Alter der SchülerInnen }\end{array}$ & - & $\begin{array}{l}\text { städtische Neue Mittelschulen, } \\
\text { Polytechnischer Lehrgang in } \\
\text { Tirol/10-15 Jahre }\end{array}$ \\
\hline Aufnahmedatum & 28.05.2019 & 26.06 .2019 \\
\hline Aufnahmedauer & 77 Minuten & 89 Minuten \\
\hline Art der Aufzeichnung & Audioaufzeichnung & Audio- und Videoaufzeichnung \\
\hline Aufnahmeort & Räumlichkeiten der SCHUSO Tirol & Räumlichkeiten der SCHUSO Tirol \\
\hline Besonderheiten & $\begin{array}{l}\text { Fragen auf Wunsch vorab } \\
\text { zugesandt }\end{array}$ & 1 Person per Skype zugeschaltet \\
\hline
\end{tabular}

Tabelle 1: Datenkorpus im Überblick

Vor allem die offene Gruppendiskussion hatte mittels gesprächsanregender Stimuli zum Ziel, diskursive Muster, kollektive Orientierungen und Praktiken offenzulegen (cf. Lüthje 2016). Bei der Gruppe handelte es sich um eine sogenannte Realgruppe (cf. Lüthje 2016), die sich in dieser Konstellation im Rahmen ihrer beruflichen Tätigkeit in regelmäßigen Abständen trifft und sich durch bereits etablierte soziale Rollen auszeichnet. Jede teilnehmende Person war darüber hinaus auch VertreterIn einer Tiroler Region, so dass den Ergebnissen der Gruppendiskussion so etwas wie regionale Repräsentativität zugesprochen werden kann. Die Räumlichkeiten, in denen die Diskussion stattfand, waren der Gruppe ebenso bekannt, so dass - mit den methodisch bedingten Einschränkungen der Aufnahme- und Interviewsituation durch eine bis dahin fremde Person - die bestmöglichen Bedingungen für ein gutes, offenes Gesprächsklima geschaffen worden waren.

Bei der Analyse bzw. kategorial offenen Kodierung und Interpretation der Daten, die mithilfe der Software FOLKER transkribiert wurden, wurde auf die qualitative Datenanalyse-Software atlas.ti zurückgegriffen. Hervorzuheben ist, dass sich die Auswertung auf metasprachliche Äußerungen bezieht, also keine natürlichen Interaktionen zwischen SchülerInnen, Eltern oder LehrerInnen mit SchulsozialarbeiterInnen analysiert werden, sondern die Schilderungen der SchulsozialarbeiterInnen über diese Gespräche im Fokus stehen. ${ }^{4}$

\section{Von den SchulsozialarbeiterInnen relevant gesetzte sprachliche Kompetenzen}

Die Daten zeigen eine hohe Sprachbewusstheit seitens der SchulsozialarbeiterInnen. Dies lässt sich zum einen erkennen an metasprachlichen Reflexionen, an Erzählungen über Gesprächs-

\footnotetext{
${ }^{4}$ Inhaltlich gingen sowohl Interview als auch Gruppendiskussion über das Thema „soziale Diversität in der Schulsozialarbeit", auf das der vorliegende Beitrag fokussiert, hinaus und behandelten etwa auch die Rolle des Beziehungsmanagements oder der interkulturellen Kommunikation.
} 
planungsprozesse und Gesprächsstrategien, Beschreibungen von für den Beruf (über den Bereich Mehrsprachigkeit hinausgehenden) vorteilhaften sprachlichen Kompetenzen, zum anderen auch an selbst kreierten Konzepten wie „sozialer dolmetsch“, womit die sprachliche Anpassung an das jeweilige Gegenüber erfasst werden soll (siehe Kapitel 4.1). Letzteres ist bereits ein Hinweis auf die sprachlichen Kompetenzen, die SchulsozialarbeiterInnen im Umgang mit sozialer Diversität benötigen.

\subsection{Das im Berufsfeld Schulsozialarbeit relevant gesetzte Sprachrepertoire}

Aus den Erzählungen der SchulsozialarbeiterInnen geht ein breites Sprachrepertoire des Sozialraums Schule hervor. In der Arbeit mit ihrer Zielgruppe kommen die SchulsozialarbeiterInnen mit SprecherInnen unterschiedlicher deutscher Varietäten (Jugendsprache, Alterssprachen (Kinder, Erwachsene), Dialekt, Umgangssprache, Standardssprache) und unterschiedlicher Sprachen in Kontakt (Deutsch als Erstsprache, Deutsch als Zweitsprache, andere Erstsprachen wie Farsi, Russisch, Somali, Syrisch-Arabisch und Türkisch, Englisch als Lingua franca). ${ }^{5}$ Diese sprachliche Diversität wird als ,größte herausforderung“ wahrgenommen, wie aus dem folgenden Zitat $^{6}$ hervorgeht:

\subsubsection{Beispiel: Experteninterview (ab min 00:41:00)}

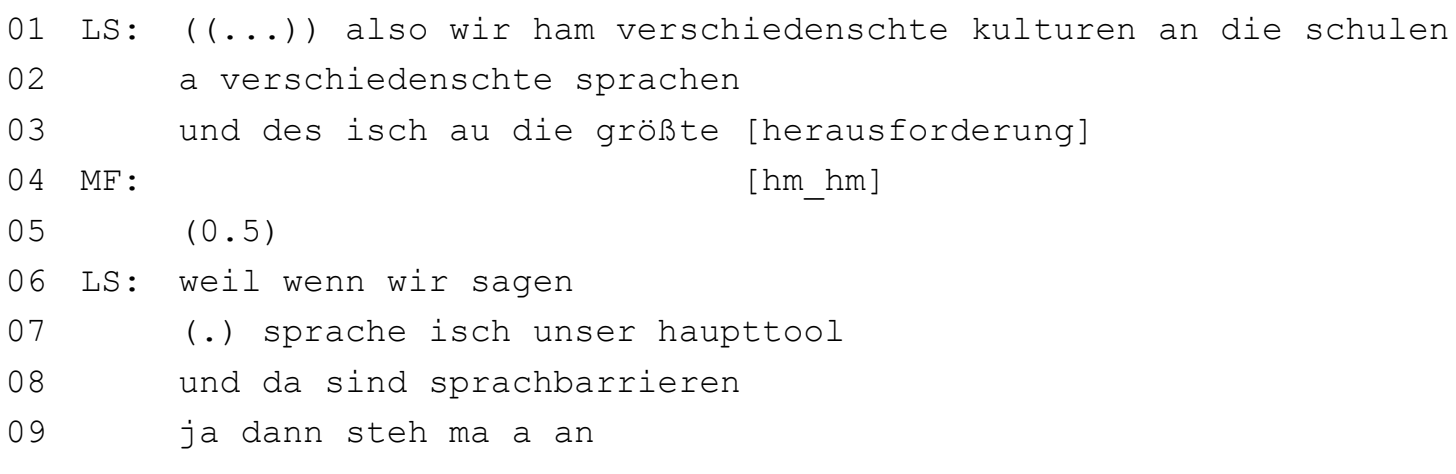

\footnotetext{
5 Der Schulstatistik der Statistik Austria zufolge sprechen in Tirol im Berichtsjahr 2018 17,6\% der SchülerInnen im Alltag hauptsächlich eine andere Sprache als Deutsch (österreichweit 26,41\%; Wien 52,18\%). Welche Sprachen genau gesprochen werden, geht aus der Schulstatistik nur begrenzt hervor: Etwas mehr als ein Drittel spricht Türkisch, ein Fünftel BKS (Bosnisch-Kroatisch-Serbisch). Die verbleibenden 44\% entfallen auf eine ,andere Sprache“, die nicht genauer ausgewiesen ist. Behelfsmäßig kann auf die Staatsbürgerschaft der SchülerInnen zurückgegriffen werden, was allerdings nur bedingt aussagekräftig sind, da viele Personen mit Migrationshintergrund über die österreichische Staatsbürgerschaft verfügen. Dennoch zeigt dieser Blick einige typische Herkunftsländer auf. 2018 verfügen insgesamt 13,18\% der SchülerInnen in Tirol über eine ausländische Staatsbürgerschaft. Die zehn häufigsten Staatsangehörigkeiten dieser SchülerInnen - exklusive der primär deutsch-, türkisch- oder BKS-sprachigen Länder - sind Italien, Syrien, Ungarn, Afghanistan, Rumänien, Russische Förderation, Irak, Bulgarien, Slowakei und Niederlande (Quelle: Schulstatistik der Statistik Austria für das Jahr 2018. Eigene Berechnungen mittels STATcube, Statistische Datenbank von Statistik Austria; Variablen: Berichtsjahr 2018, Umgangssprache nach Bundesland, Staatsangehörigkeit nach Bundesland).

${ }^{6}$ Da die Daten in diesem Beitrag inhaltsanalytisch betrachtet werden, werden Zitate aus den Befragungen in Form von Minimaltranskripten nach GAT 2 wiedergegeben. Die Kürzel in den Transkripten stehen für folgende Personenbezeichnungen: $\mathrm{EI}=$ Experteninterview, GD $=$ Gruppendiskussion, $\mathrm{MF}=$ Marianne Franz, LS $=$ Leiter der Schulsozialarbeit, SO1/SO2/SO3/SO4/SO5 = befragte SozialarbeiterInnen der Gruppendiskussion. Die Gespräche wurden überwiegend im Nonstandard geführt. MF und SO2 bis SO5 sprechen im Südbairischen und Südmittelbairischen angesiedelte Varietäten, SO1 eine Varietät mit alemannischem Einfluss (cf. Scheuringer 1997).
} 
Im Umgang mit der sprachlichen Diversität ihrer Zielgruppe erweisen sich ihren Schilderungen zufolge produktive Kompetenzen in Dialekt, Umgangssprache, Standardsprache und Fachsprache sowie in Fremdsprachen, vor allem Englisch (als Lingua franca), erwähnt wird auch Italienisch, als hilfreich. Die SchulsozialarbeiterInnen wählen aus ihrem Repertoire sehr bewusst und wissen, dass verschiedene Situationen den Einsatz verschiedener sprachlicher Varietäten erfordern. Gerade beim Gespräch mit Jugendlichen sind jugendsprachliche Kompetenzen von Vorteil - allerdings rein rezeptiv, eine aktive Verwendung von jugendsprachlichen Kompetenzen wird als nicht authentisch und anmaßend empfunden. Ähnliches gilt für andere Dialekte, die sie nicht sprechen, aber verstehen müssen. Dennoch reicht das Sprachrepertoire der SchulsozialarbeiterInnen angesichts der sprachlichen Diversität des Sozialraums Schule nicht aus und es treten „sprachbarrieren“ (Beispiel 3.1.1, Seg. 08) auf, die die Arbeit erschweren und die SchulsozialarbeiterInnen ,,anstehen“ lässt (Seg. 09).

\subsection{Soft communicative skills oder: Diversitätskompetenz}

Als besonders bedeutsam im Umgang mit sozialer und sprachlicher Diversität werden in den Daten Einstellungen und Handlungskompetenzen konstruiert, die mit Wilde et al. (2018: 396) als soft communicative skills beschrieben werden können: ,[...] 'hard' language difference is imagined as surmountable by 'soft' communicative skills, such as using clear, plain language and adopting an open communicative atittitude".

Die SchulsozialarbeiterInnen gehen zwar nicht davon aus, dass mit diesen soft communicative skills harte Sprachbarrieren tatsächlich überwunden werden können (siehe Kapitel 5), aber sie halten sie dennoch für geeignet, verschiedenartigste Sprachbarrieren, die die innere und äußere Mehrsprachigkeit betreffen, abzuschwächen, weshalb sie zu den sprachlichen Kernkompetenzen der Schulsozialarbeit zählen. Dazu gehören zum einen

- die Fähigkeit zur Selbstreflexion in Zusammenhang mit einem Bewusstsein der eigenen Kulturgebundenheit (siehe Beispiel 3.2.1),

- das Wissen über andere Kulturen und kulturell bedingte Sprachnormen z. B. in Bezug auf Höflichkeitsnormen (muslimischer Schüler hält keinen Blickkontakt bei einer Rüge durch den Lehrer, EI ab min 00:44:10; ein bzw. eine SchulsozialarbeiterIn wäre im ,idealfall“ auch ,ethnologe oder ethnologin“, EI ab min 00:47:53),

- Toleranz gegenüber anderen Kulturen und auch Sprachen bzw. Varietäten (GD ab min 00:19:11),

- das „interesse“ für Kinder und Jugendliche, deren Themen und deren Sprache (etwa Jugendsprache) (EI ab min 01:07:08) sowie

- Empathie bzw. „fingerspitzengefühl“" (EI ab min 01:06:36).

\subsubsection{Beispiel: Experteninterview (ab min 00:43)}

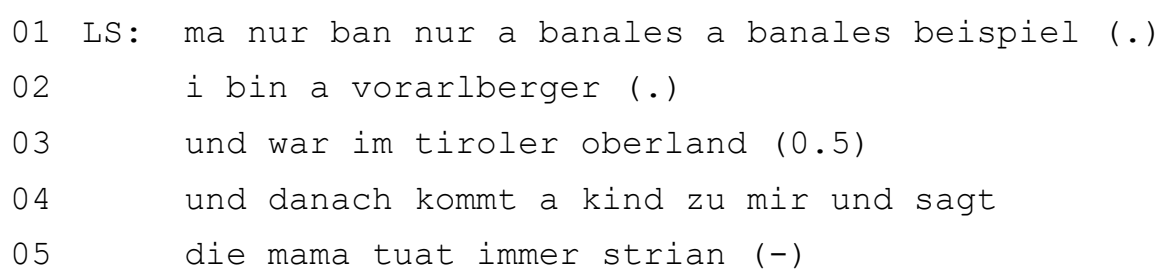




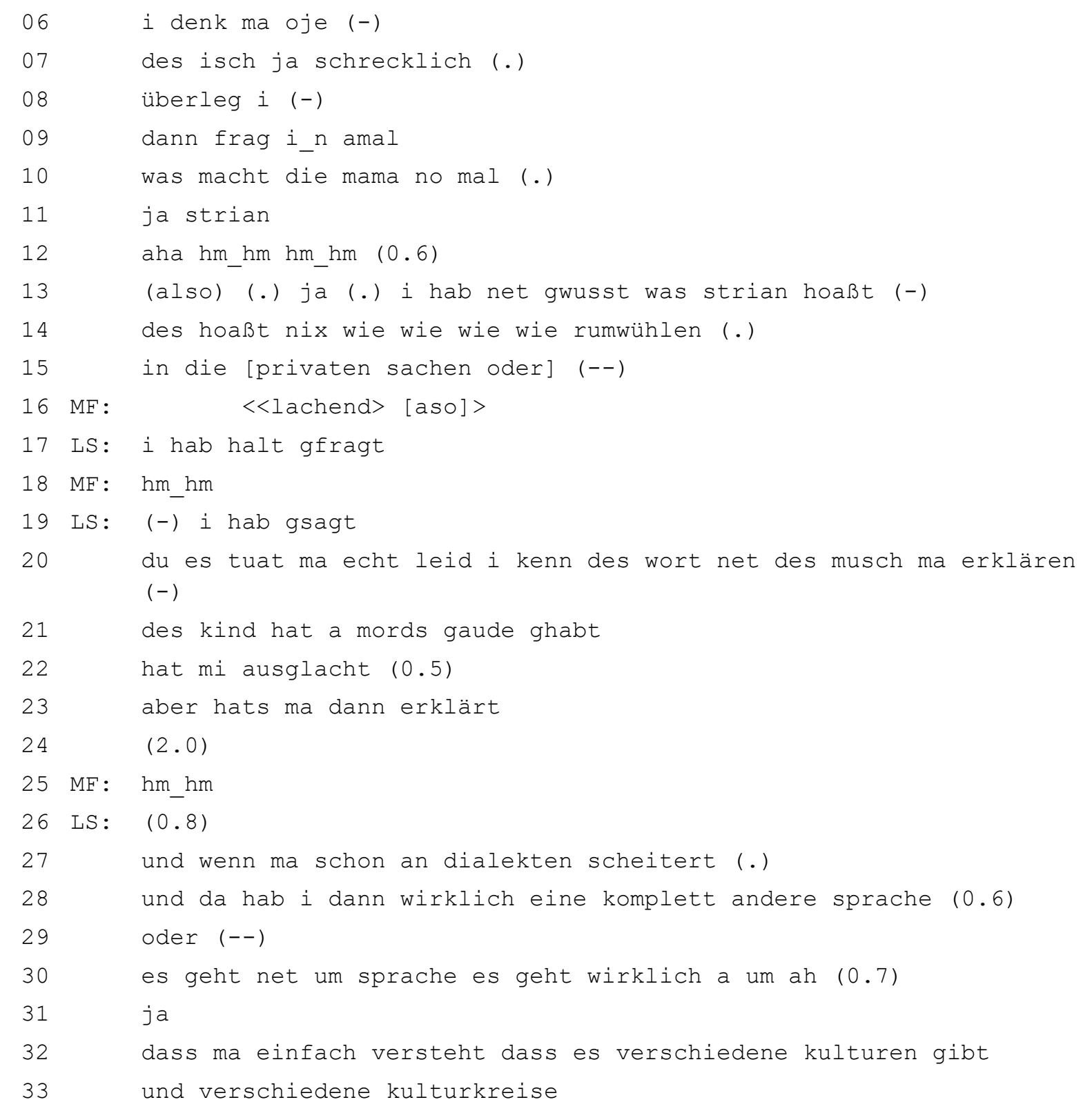

In Beispiel 3.2.1 zeigt sich die offene kommunikative Haltung des Schulsozialarbeiters im Nachfragen bzgl. eines vom Schüler verwendeten dialektalen Wortes, das er nicht verstanden hat (Seg. 1-23), aber auch in einer Haltung der Selbstreflexion bzgl. der eigenen Kultur und der Kultur des Gegenübers (Seg. 32-33).

Die von den SchulsozialarbeiterInnen beschriebenen Kompetenzen können überzeugend mit dem Modell der drei Komponenten interkultureller Kompetenz nach Erll/Gymnich (2011) in Verbindung gebracht werden, ein Vergleich, der angesichts der sozialen und in diesem Zusammenhang auch kulturellen Diversität des Sozialraums Schule durchaus angebracht ist. Kultur selber verstehen die SchulsozialarbeiterInnen im weiten, d. h. anthropologischen Sinne, am besten beschreibbar mit dem Begriff des Kollektivs bei Hansen (2011). Darunter sind identitätsund wertestiftende, dynamisch-hybride (cf. Reimann 2017: 20), interaktional ausgehandelte (cf. Spitzmüller 2017) (Mehrfach-)Zugehörigkeiten zu einer bzw. mehreren Gemeinschaft(en) zu verstehen. Das können jugendliche Subkulturen genauso sein wie Stadtteilkulturen, dörfliche Vereinskultur sowie religiös oder ethnisch geprägte Kulturen (cf. auch die Ausführungen in 
Franz i. V.). Interkulturelle Kompetenz ist den Erzählungen der SchulsozialarbeiterInnen entsprechend nicht nur hilfreich in den Gesprächen mit Kindern und Erwachsenen mit Migrationshintergrund, und könnte hier insofern besser mit Diversitätskompetenz bezeichnet werden (cf. Arnold 2018), als dass dieser Begriff den Fokus auf ganz unterschiedlich geartete Diversität legt, sodass auch andere Unterschiede mitgemeint sein können - etwa das unterschiedliche Alter der Kinder mit den damit verbundenen unterschiedlichen kognitiven und sprachlichen Fähigkeiten. Abbildung 1 zeigt nun die Zusammenführung der Erzählungen der SchulsozialarbeiterInnen (Außenkreis) mit dem Modell der interkulturellen Kompetenz nach Erll/Gymnich (2011; zit. nach Reimann 2017: 27-28) (die beiden inneren Kreise).

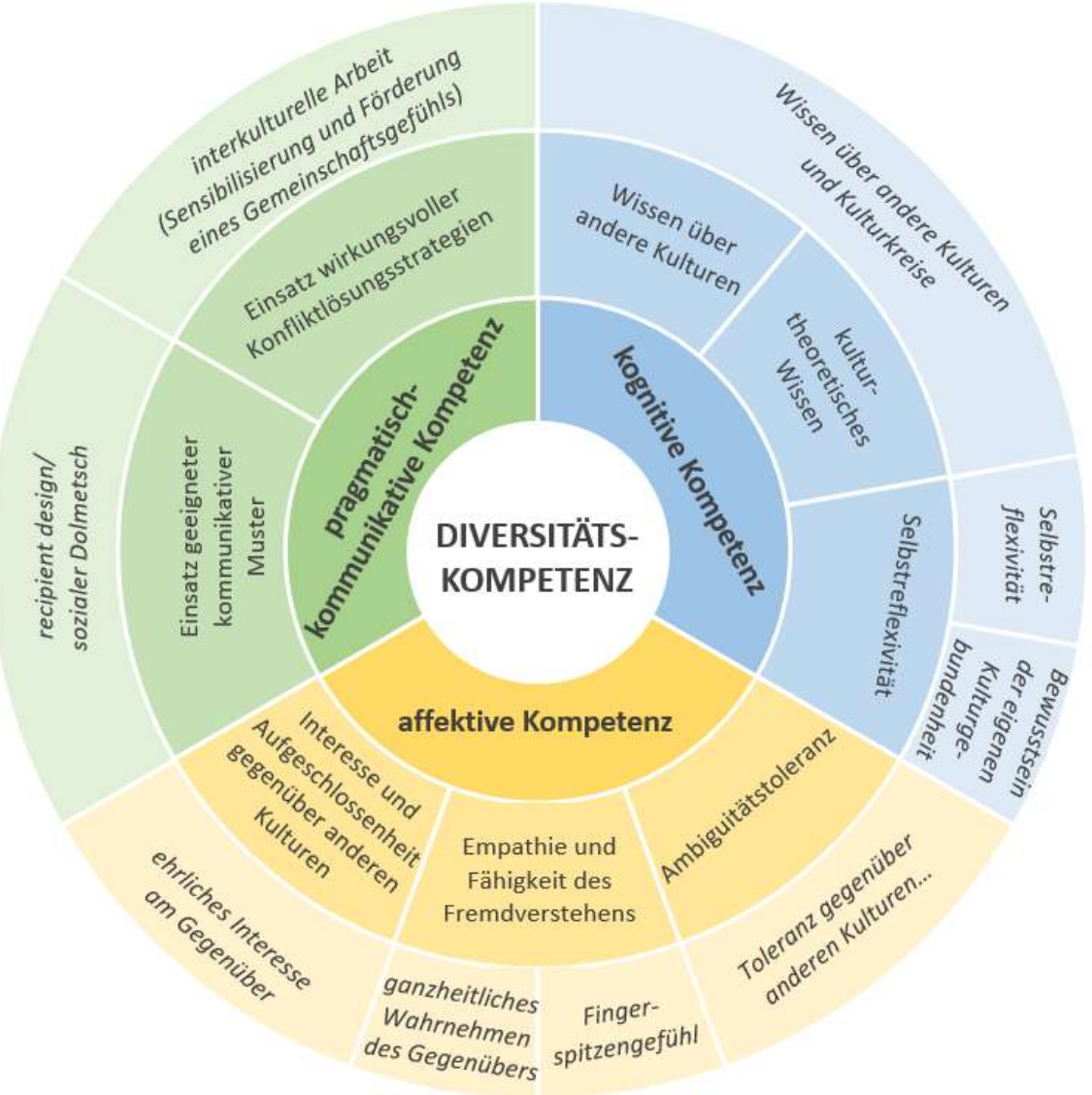

\section{Abbildung 1: Diversitätskompetenz in der Schulsozialarbeit in Anlehnung an das Modell interkultureller Kompetenz nach Erll/Gymnich 2011 (eigene Darstellung)}

Die in Abbildung 1 dargestellten Kompetenzen helfen den SchulsozialarbeiterInnen, interkulturelle hotspots (Situationen, die Missverständnisse produzieren) oder auch rich points (Situationen, die Sprach- und Kulturbewusstsein evozieren) (cf. Agar 1994, zitiert nach Neuland 2013: 171-172) zu erfassen und entsprechend zu agieren. Sie erleichtern die Kommunikation in einem sozial, sprachlich und kulturell diversen Umfeld, erweitern den Handlungsspielraum und erhöhen die Wahrscheinlichkeit des Gesprächsgelingens. Hinsichtlich der pragmatischkommunikativen Kompetenz ist abseits des recipient designs selbst (siehe Kapitel 4.1) noch anzuführen, dass die SchulsozialarbeiterInnen ihre Aufgabe auch darin sehen, im Sozialraum Schule interkulturelle Arbeit zu leisten und im Sinne einer Kulturmittlerschaft diese interkultu- 
relle Kompetenz auch seitens der SchülerInnen und LehrerInnen zu fördern (Bsp. interkulturelles Frühstück - GD ab min 00:42:49; kritische Reflexion des von einer Lehrperson mit ihrer Klasse vereinbarten Türkischverbots in der Pause - GD ab min 00:45:44).

\section{Kommunikative Praktiken der SchulsozialarbeiterInnen im Umgang mit sprach- licher Diversität}

Unter den verschiedenen kommunikativen Praktiken, die die SchulsozialarbeiterInnen einsetzen, um mehrsprachige Interaktionen zu bewältigen, sind nur wenige Top-down-Praktiken zu finden. Diese beschränken sich auf die Kompensation fehlender Fremdsprachenkenntnisse der SchulsozialarbeiterInnen durch extern (mit entsprechender logistischer Vorlaufszeit) anzufordernde DolmetscherInnen. Punktuell wurde versucht, auch SchulsozialarbeitInnen mit Migrationshintergrund und entsprechenden Sprachkenntnissen zu verpflichten - eine Praxis, von der aufgrund der großen sprachlichen Diversität innerhalb des Sozialraums Schule bald wieder abgesehen wurde: Ein Migrationshintergrund allein reicht nicht aus, um die gesamte sprachliche Diversität abzudecken (EI ab min 00:42:44). Hinsichtlich der inneren Mehrsprachigkeit werden keine Top-down-Praktiken angeführt. Der Umgang damit wird im Sinne des learning by doing im Beruf angeeignet. So wird festgestellt, dass gerade, wenn man frisch von der Ausbildung in den Beruf einsteigt, das fachsprachlich Erlernte übersetzt werden muss und der Wechsel hin zu einer kindgerechten Sprache nicht immer gleich gelingt (GD ab min 01:12:34; EI ab min 00:39:40).

Überhaupt ist es der berufliche Alltag, der die SchulsozialarbeiterInnen dazu drängt, im Umgang mit Mehrsprachigkeit eigenständige Lösungen zu finden, so dass Kommunikation möglich wird. Diese Praktiken reichen von recipient design (cf. Sacks/Schegloff/Jefferson 1974) über muldimodale bis hin zu multimedialen Praktiken. Auf alle Praktiken soll in den nun folgenden Kapiteln im Detail eingegangen werden.

\section{1 sozialer dolmetsch oder: recipient design}

Die SchulsozialarbeiterInnen wählen aus ihrem Sprachrepertoire in Abhängigkeit ihres Gegenübers die ihrer Ansicht nach passend(st)e Sprache bzw. Varietät aus, was im Experteninterview auch als sozialer dolmetsch bezeichnet wird:

\subsubsection{Beispiel: Experteninterview (ab min 00:39:02)}

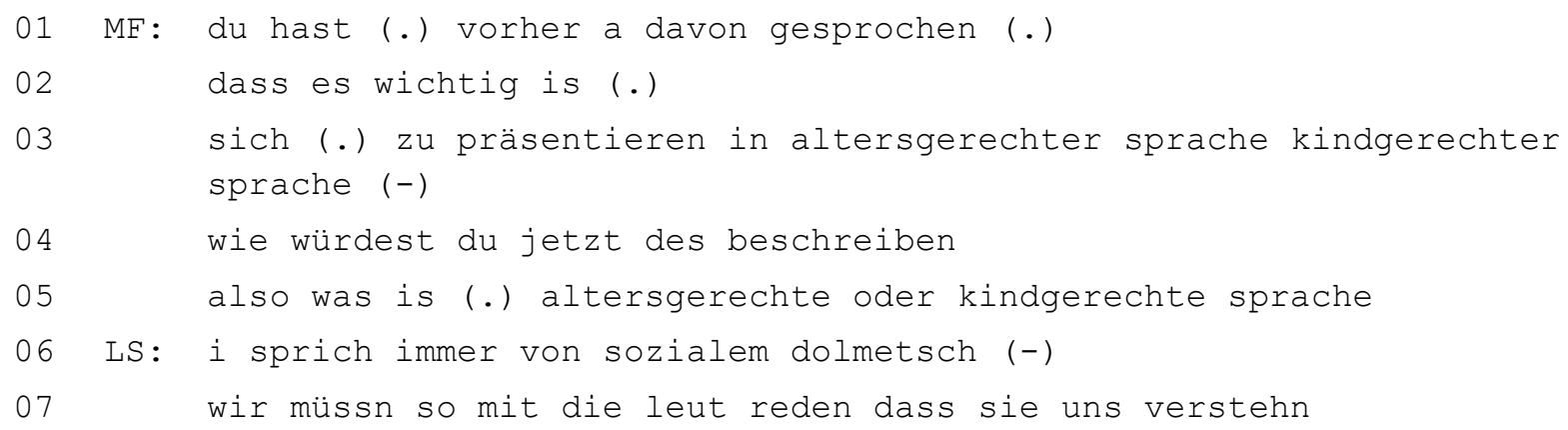

In der Linguistik hat sich für dieses interaktionale Verfahren das Konzept des recipient design (cf. Sacks/Schegloff/Jefferson 1974) etabliert. Schmitt/Deppermann (cf. 2009: 82) zufolge 
kennzeichnet es sich durch drei Merkmale: (1) Das recipient design beruht auf Annahmen der SprecherInnen über ihre GesprächspartnerInnen, etwa hinsichtlich deren Wissensbestände. (2) Die sprachlichen Anpassungsverfahren des recipient design sind beobachtbar. (3) Recipient design wird interaktiv gestaltet und ausgehandelt.

Maßgeblich für die Ausgestaltung des recipient designs sind demnach Partnerhypothesen und Fremdpositionierungen der SchulsozialarbeiterInnen, d. h. wie sie ihre GesprächspartnerInnen einschätzen und auch deren Identitäten, deren Wissen oder Emotionen im Gespräch positionieren (cf. Schmitt/Deppermann 2009: 106). Die SchulsozialarbeiterInnen selbst führen folgende Faktoren an, die die Art ihres ,sozialen dolmetsch“ beeinflussen (Tabelle 2):

\begin{tabular}{|c|c|}
\hline Fremdpositionierungen/Partnerhypothesen & Selbstpositionierungen \\
\hline $\begin{array}{l}\text { - Annahmen über das Verstehen bei den Rezipien- } \\
\text { tInnen } \\
\text { - } \\
\text { soziolinguistische Eigenschaften der Rezipien- } \\
\text { tInnen wie Alter, Geschlecht, Rolle (GD ab min } \\
\text { 01:00:24), Sprachrepertoire (GD ab min } \\
\text { 01:13:29) } \\
\text { - Annahmen über das emotionale Befinden der } \\
\text { RezipientInnen }\end{array}$ & 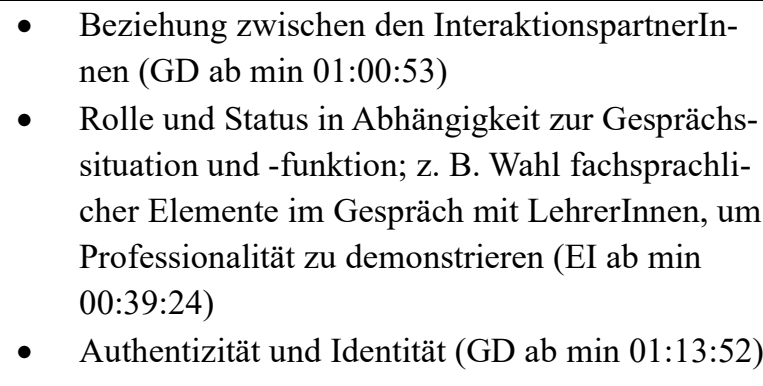 \\
\hline
\end{tabular}

Tabelle 2: Recipient design beeinflussende Fremd- und Selbstpositionierungen

Neben den Fremdpositionierungen wirken auch die Selbstpositionierungen der SchulsozialarbeiterInnen auf das recipient design ein - v. a. wenn es darum geht, die eigenen personalen und sozialen Identitäten und Zugehörigkeiten diskursiv zu konstruieren (cf. Deppermann 2013: Abs. 46) oder die eigenen Rollen zu etablieren. In der Schulsozialarbeit besonders relevant ist auch das Beziehungsmanagement bzw. ,relational work“ (von Locher/Watts (2008: 78) definiert als ,work people invest in negotiating their relationships in interaction“), das zwischen den GesprächspartnerInnen getätigt wird.

In engem Zusammenhang mit dem recipient design stehen die expliziten und impliziten Verstehensthematisierugen. Die ProbandInnen streichen diesbezüglich nicht nur die Bedeutung des Zuhörens hervor, sondern auch die Kompetenz des multimodalen Wahrnehmens des Gegenübers bzgl. impliziter Verstehenssignale: Wie reagiert er/sie? Was zeigt seine/ihre Körperhaltung? Auch mittels Rückfragen werden explizite Verstehensthematisierungen eingefordert (z. B. EI ab min 00:55:04). Diese sogenannten Verstehensdokumentationen (cf. Deppermann/Schmitt 2008; Imo 2013) geben den SprecherInnen Hinweise auf die Wissensbestände und das Verstehen der GesprächspartnerInnen, so dass die SprecherInnen entsprechend ihre sprachlichen Äußerungen konstruieren, adaptieren oder korrigieren bzw. reformulieren können (siehe Beispiel 5.1).

Abseits der Faktoren, die das recipient design beeinflussen, interessiert natürlich, auf welche Weise bzw. mit welchen sprachlichen Mitteln recipient design überhaupt geschieht. Sacks/Schegloff/Jefferson (cf. 1974: 727; zit. nach Schmitt/Deppermann 2009: 81) führen hier etwa Wort- und Themenwahl, die Ausgestaltung der Gesprächssequenzen oder der Gesprächseröffnung und -beendigung an. Den SchulsozialarbeiterInnen zufolge geschieht ihre Anpassung 
ans Gegenüber auf verschiedenen sprachlichen Ebenen und betrifft sowohl die innere als auch die äußere Mehrsprachigkeit:

- Anpassung der Wortwahl

- Anpassung der Syntax (z. B. einfachere Syntax im Gespräch mit Kindern)

- Anpassung der Themenwahl

- Anpassung des Sprechtempos

- Wahl einer bestimmten Varietät (Dialekt, Umgangssprache, Standardsprache)

- Wahl einer bestimmten Sprache (Fremdsprache, z. B. Englisch als Lingua franca)

\subsection{Dolmetschen}

Gibt es keine gemeinsame Sprache mit SchülerInnen oder Erziehungsberechtigten, wird wenn möglich auf Dolmetschdienste zurückgegriffen. Im Kontext einer öffentlichen bzw. sozialen Einrichtung wie es auch die Schulsozialarbeit ist, spricht man von sogenanntem „community interpreting“" (zum Begriff cf. Pöllabauer 2005: 49-62). Dieses zeichnet sich dadurch aus, dass die sprachmittelnde Person Face-to-Face-Interaktionen bidirektional, also in beide Sprachrichtungen, zu dolmetschen hat.

Grundsätzlich ist im Kontext der Schulsozialarbeit zwischen Praktiken des professionellen Dolmetschens und Praktiken des Laiendolmetschens zu unterscheiden. Professionelles Dolmetschen wird über verschiedene Institutionen wie die Bildungsdirektion oder die Kinder- und Jugendhilfe organisiert und finanziert, wobei die finanziellen Mittel den Aussagen der SchulsozialarbeiterInnen zufolge beschränkt sind. Teilweise werden DolmetscherInnen auch per Videotelefonie konsultiert. Dolmetsch-Bedarf ist jeweils frühzeitig anzumelden, was den Handlungsspielraum der SchulsozialarbeiterInnen stark einschränkt, da mit einer entsprechenden Vorlaufszeit gerechnet werden muss und spontane Gespräche mit den betroffenen SchülerInnen oder Eltern - eine der Stärken der Schulsozialarbeit - nur schwer möglich sind (EI ab min 00:41:34). Dieser strukturelle Kontext bewirkt, dass immer wieder auch auf LaiendolmetscherInnen, also Personen ohne professionelle Sprachmittlungsausbildung, rekurriert wird. Zum Teil sind diese institutionell und von öffentlicher Hand finanziert. Erwähnt wird hier das sogenannte „Mobile interkulturelle Team“, das vom Bildungsministerium aus dem sogenannten Integrationstopf finanziert wird und dessen primäre Aufgabe in der Betreuung und Integrationsförderung kürzlich in Österreich angekommener MigrantInnen bzw. Flüchtlingskinder besteht (cf. BMBF 2016). Das Team arbeitet überregional und besteht aus einem Mix aus SozialpädagogInnen, SozialarbeiterInnen und PsychologInnen mit spezifischen Sprachkenntnissen. Den Aussagen der befragten SchulsozialarbeiterInnen zu Folge wird dieses Team von ihnen gerade aufgrund dieser sprachlichen Kompetenzen angefordert (GD ab min 00:32:34; EI ab min 00:51:11), obwohl Dolmetschen im Grunde nicht zum definierten Tätigkeitsbereich des „Mobilen interkulturellen Teams“ zählt und dieses auch über keine entsprechende Ausbildung verfügt (cf. die Aufgabenbeschreibung in BMBF 2016).

Als LaiendolmetscherInnen fungieren aber insbesondere auch Personen, die dem jeweiligen Sozialraum Schule angehören und über entsprechende sprachliche Kompetenzen verfügen. Das können schulinterne Personen wie SchülerInnen oder LehrerInnen (GD ab min 01:16:43; GD ab min 00:29:10), aber auch schulexterne Personen wie etwa die Betreuerin eines SchülerInnen- 
Wohnheims (GD min 00:31:59) sein. Die SchulsozialarbeiterInnen greifen damit auf die „kollektive Mehrsprachigkeit“" (cf. Dannerer/Franz 2019: 269) der Schulgemeinschaft zurück. Der Vorteil dieser Herangehensweise liegt im geringeren organisatorischen und zeitlichen Aufwand. Welche Herausforderungen mit LaiendolmetscherInnen verbunden sind, wird in Kapitel 5 ausgeführt.

\subsection{Multimodale Praktiken}

Hierunter sind Praktiken zu verstehen, die die verbalen Handlungen begleiten und unterstützen. Dies sind zum einen para- und nonverbale Handlungen, die den Körper als interaktive Ressource nutzen, zum anderen aber auch der begleitende Einsatz von räumlichen Ressourcen wie dem im Büro der SchulsozialarbeiterInnen vorhandenen Material (cf. Hausendorf/Mondada/Schmitt 2012). ${ }^{7}$

Nonverbales kann als von den SchulsozialarbeiterInnen bei ihren GesprächspartnerInnen wahrgenommenes Verstehenssignal (EI min 00:55:04), aber auch im Sinne des ,mit händen und füßen“-Sprechens (EI min 00:54:07) helfen, zu einer Verständigung zu kommen. Das kommunikative Handeln im eigenen Büro wird hier als vorteilhafter gegenüber einem Klassenzimmer angesehen (cf. auch Kapitel 4.5). Dies rührt von der 1:1-Konstellation eines Einzelgesprächs, das es erlaubt, in intensiven Austausch mit einer einzelnen Person zu kommen - im Gegensatz zur Arbeit mit einer großen Gruppe im Klassenzimmer im Rahmen eines Elternabends oder einer Präventionseinheit mit einer Klasse, wo Verstehensmanifestationen nur sehr eingeschränkt möglich und wahrnehmbar sind (GD min 01:15:40).

\subsection{Multimediale Praktiken}

Eine weitere Praktik, mit Mehrsprachigkeit umzugehen, steht mit den räumlichen Ressourcen in Zusammenhang, zielt aber nicht auf eine andere Modalität neben Sprache ab, sondern auf eine andere Medialität der Sprache: Es handelt sich um den Einsatz von Schriftlichkeit, um Schwierigkeiten im mündlichen Gespräch zu entgegnen. Dies kann eine in englischer Sprache verfasste E-Mail an Eltern sein (GD ab min 00:29:04) ebenso wie digitale bzw. internetbasierte Übersetzungshilfen wie der, ,google translator“, die während eines Einzelgesprächs mit einer Schülerin oder einem Schüler mit einer anderen Erstsprache als Deutsch zum Einsatz kommen (EI ab min 00:53:15, EI ab min 00:53:56, GD ab min 00:28:05). Diese Übersetzungshilfen ermöglichen zwar einen gewissen Grad an Kommunikation (EI ab min 00:53:51), können aber das Fehlen geteilter Sprachkompetenzen nicht gänzlich kompensieren, so dass komplexere Themen nicht behandelt werden können (GD ab min 00:28:19: ,da (.) siegt man sich dann bei ganz basale sachen ah zum erklären ahm weil (--) höher gehts dann nicht mehr ge“").

\footnotetext{
${ }^{7}$ Der Raum wird nicht nur in mehrsprachigen Gesprächen als Ressource genutzt. Die SchulsozialarbeiterInnen erzählen von einem Büro in einer Volksschule, in dem auch eine Sand- oder Reiskiste für die Kinder zur Verfügung steht (EI min 00:58:47). Das Spielen mit diesen Materialien erleichtert es den Kindern, mit den SchulsozialarbeiterInnen parallel ins Gespräch zu kommen, und mindert kommunikative Hemmschwellen. Auch gestalterisches Arbeiten (Zeichnen, Auflegen von Legematerial; GD min 00:09:52) oder gemeinsames Spazieren (EI min 00:11:04) (cf. auch Haddington/Mondada/Nevile 2013) werden als interaktive Angebote gesetzt.
} 


\section{$5 \quad$ Bleibende Herausforderungen}

In Kapitel 4 wurden vielfältige Top-down- und Bottom-up-Praktiken beschrieben, die die Arbeit der SchulsozialarbeiterInnen mit ihrer mehrsprachigen Zielgruppe anwenden. Obwohl den SchulsozialarbeiterInnen zufolge vieles funktioniert und ein Teil mehrsprachiger Gespräche durchaus als erfolgreich klassifiziert wird, berichten die Befragten auch von zahlreichen ungelösten Herausforderungen im Zusammenhang mit Mehrsprachigkeit. Diese Herausforderungen betreffen insbesondere „harte Sprachbarrieren“ (Wilde et al. 2018: 396) und gedolmetschte Gespräche.

Harte Sprachbarrieren treten zutage, wenn keinerlei oder nur sehr geringe Deutschkenntnisse seitens der AdressatInnen und auch keine gemeinsame Lingua franca vorhanden sind. Diese harte Sprachbarriere bedeutet letztlich eine Zugangsbarriere zum Angebot der Schulsozialarbeit. Bestimmte Gruppen können nicht oder nur schlecht erreicht werden. Auch das folgende Zitat demonstriert das kommunikative Ringen der SchulsozialarbeiterInnen in solchen Interaktionskonstitutionen:

\subsection{Beispiel: Gruppendiskussion (ab min 00:26:24)}

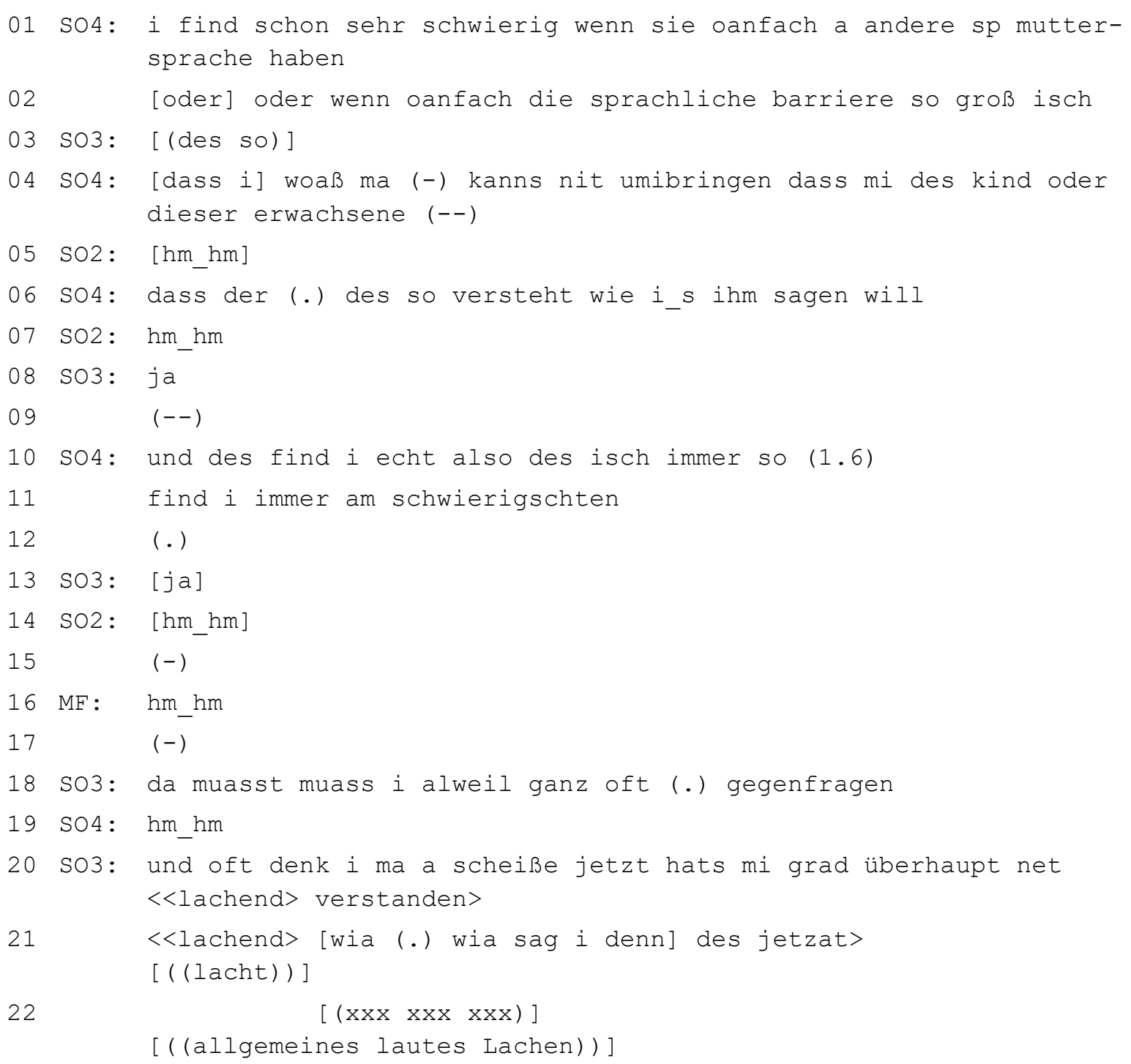




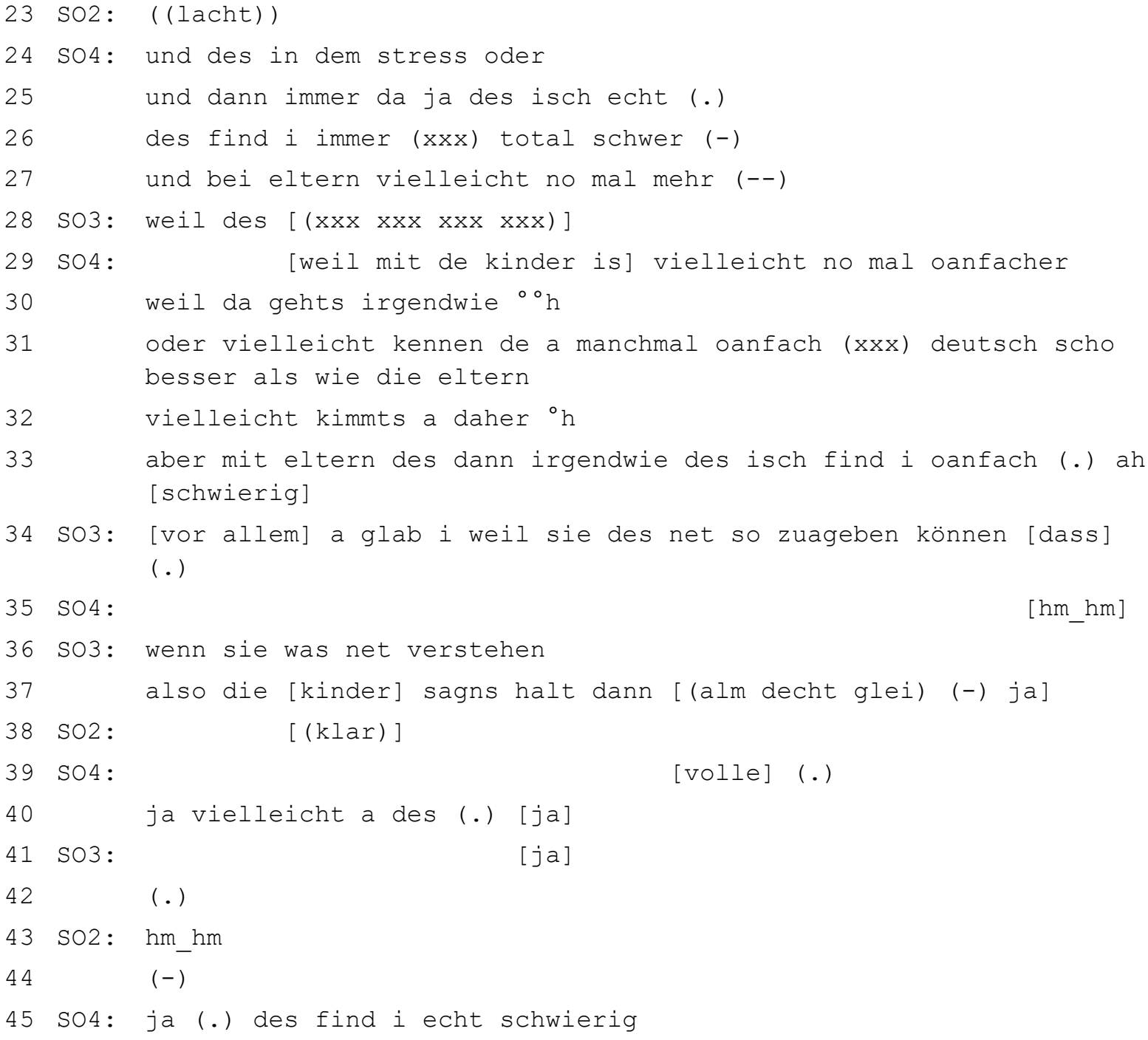

Die Schulsozialarbeiterin versucht durch ,gegenfragen“ (Seg. 18), also Verstehensthematisierungen, ebenso wie Reformulierungen (,wia sag i denn des jetzat“", Seg. 21) Verständnis bei dem/der InteraktionspartnerIn zu erzielen, und räumt ein, mitunter zu scheitern (,scheiße jetzt hats mi grad überhaupt net verstanden“, Seg. 20). Erschwerend tritt hinzu, dass Verstehensthematisierungen auch deshalb mitunter erfolglos bleiben, weil sich GesprächspartnerInnen als sprachlich kompetent positionieren und Verständnis lediglich vortäuschen. Zuzugeben, „wenn sie was net verstehen“ (Seg. 36), wird von den SchülerInnen manchmal, stärker aber von den Eltern („,bei eltern vielleicht no mal mehr“, Seg. 27) als gesichtsbedrohender Akt empfunden (cf. Imo 2013: 292), den es zu vermeiden gilt (auch EI ab min 00_55_04). ${ }^{8}$ Überhaupt erleben die SchulsozialarbeiterInnen das recipient design als herausfordernd - nicht nur in Bezug auf Personen mit geringen Deutschkenntnissen, sondern gernerell im Gespräch mit Kindern, wo es gilt, die Wörter so zu wählen, dass die SchulsozialarbeiterInnen auch verstanden werden (GD ab min 00:27:32).

\footnotetext{
${ }^{8}$ Interaktionsanalytisch auffallend sind hier die Sprecherwechsel im Zusammenhang mit den vielen zustimmenden Rezeptionssignalen verschiedenster SprecherInnen, die darauf hinweisen, dass alle befragten SchulsozialarbeiterInnen hier ähnliche Erfahrungen gemacht zu haben scheinen.
} 
Professionelle Dolmetschdienste, die dabei helfen könnten, Sprachbarrieren aufgrund einer fehlenden gemeinsamen Sprache zu überwinden, sind nur eingeschränkt verfügbar, bedürfen einer längeren Vorlaufszeit und einer entsprechenden Finanzierung. Der Handlungsspielraum der SchulsozialarbeiterInnen ist dadurch stark begrenzt; spontanes und flexibles Handeln, das die Schulsozialarbeit auch auszeichnet (Gespräche am Gang, spontan entstehende Einzelgespräche im Büro), ist nicht möglich. Hinzu kommt das zum Teil geringe Sprachkapital allochtoner Eltern in ihrer L1 (cf. Brizić 2007), was die Kommunikation (sei es in einer Fremdsprache, sei es vermittelt durch eine/n DolmetscherIn) noch zusätzlich erschwert (GD ab min 00:31:12). Wenn die Sprachbarrieren derart hoch sind, sind die SchulsozialarbeiterInnen gezwungen, sich auf bestimmte Themen zu beschränken (Konfliktmoderation geht noch, aber Schwierigkeiten in der Familie nicht - GD ab min 00:29:51) oder zumindest die sprachliche und inhaltliche Komplexität massiv zu reduzieren, wodurch jedoch Aspekte und Möglichkeiten verloren gehen. Können gewisse Themen nicht oder nicht gut besprochen werden, gewisse Zielgruppen nicht oder nur schlecht erreicht werden, steht dies im Gegensatz zum Kernanliegen und -aufgabenfeld der SchulsozialarbeiterInnen, für alle SchülerInnen da zu sein. Auch ist es gerade die politisch anivisierte Zielgruppe der SchülerInnen mit Migrationshintergrund (bzw. deren Eltern eine andere Erstsprache als Deutsch haben; siehe Kapitel 1), die schwerer erreicht wird. Die SchulsozialarbeiterInnen ringen hier nach Wegen und Lösungen, Sprachbarrieren zu überwinden, und wünschen sich einschlägige Fortbildungen (EI ab min 01:08:17).

Auch gedolmetschte Gespräche bringen Herausforderungen mit sich. Das Dolmetschen wird von den SchulsozialarbeiterInnen zum einen zwar als Erleichterung und Arbeitsersparnis dargestellt (GD ab min 00:30:43), zum anderen aber sehr kritisch gesehen. Das ursprüngliche Zweier-Gespräch wird durch die sprachmittelnde Person zu einem triadischen Gespräch. Die DolmetscherInnen beeinflussen die Interaktion nicht nur durch ihre räumlich (sehr nahe) Anwesenheit, sondern ko-konstruieren das Gespräch auch aktiv in wechselnden Partizipationsrollen mit (cf. Kadrić 2016: 104-105): Sie koordinieren metakommunikativ die Gespräche, sie steuern Sprecher- oder Themenwechsel, erläutern Hintergründe (cf. auch das Konzept des language und cultural brokers, z. B. bei Hlavac 2014), lassen Überflüssiges weg (cf. gatekeeping im Dolmetschen, z. B. bei Davidson 2000). Auf welche Weise sie das tun, hängt maßgeblich von ihrem Rollenverständnis und auch von ihrem Professionalisierungsgrad ab. Community interpreting ist eine komplexe Tätigkeit, die weit über die reine Wiedergabe des Gesagten hinausgeht und maßgeblich zum Gelingen oder Misslingen des Gesprächs beiträgt (cf. Kadrić 2016: 104-105). Es ist fragwürdig, inwieweit sich hinzugezogene LaiendolmetscherInnen dieser Prozesse bewusst sind. Hinzu treten potentielle Rollen-, Status- und Interessenskonflikte, aber auch moralische und ethische Dilemmata gerade bei Kindern, die ihre Eltern, ihre Geschwister (siehe Beispiel 5.2, Seg. 7-11) oder ihre SchulkollegInnen dolmetschen (cf. hier auch die Ausführungen in Zwengel 2015 für den Schulbereich sowie für einen allgemeinen Überblick Morales/Hanson 2005). Die SchulsozialarbeiterInnen sehen das Dolmetschen durch Kinder v. a. bei Einzelberatungen sehr kritisch, weil diese Situationen die Kinder überfordern (EI min ab 00:41:51) und weil Kinder hier oft in einen inneren Konflikt geraten (siehe Beispiel 5.2: „was übersetzens denn jetzt überhaupt genau“, Seg. 5). Diese Überforderungssituation wurde in der Sprachmittlungsforschung auch schon für das Familien-Dolmetschen in medizinischen 
Kontexten festgestellt (cf. z. B. Kletečka-Pulker/Parrag 2018: 57). Trotzdem sind die SchulsozialarbeiterInnen in ihrer Arbeit auf das Dolmetschen durch Kinder und erwachsene Laien angewiesen.

\subsection{Beispiel: Gruppendiskussion (ab min 00:30:20)}

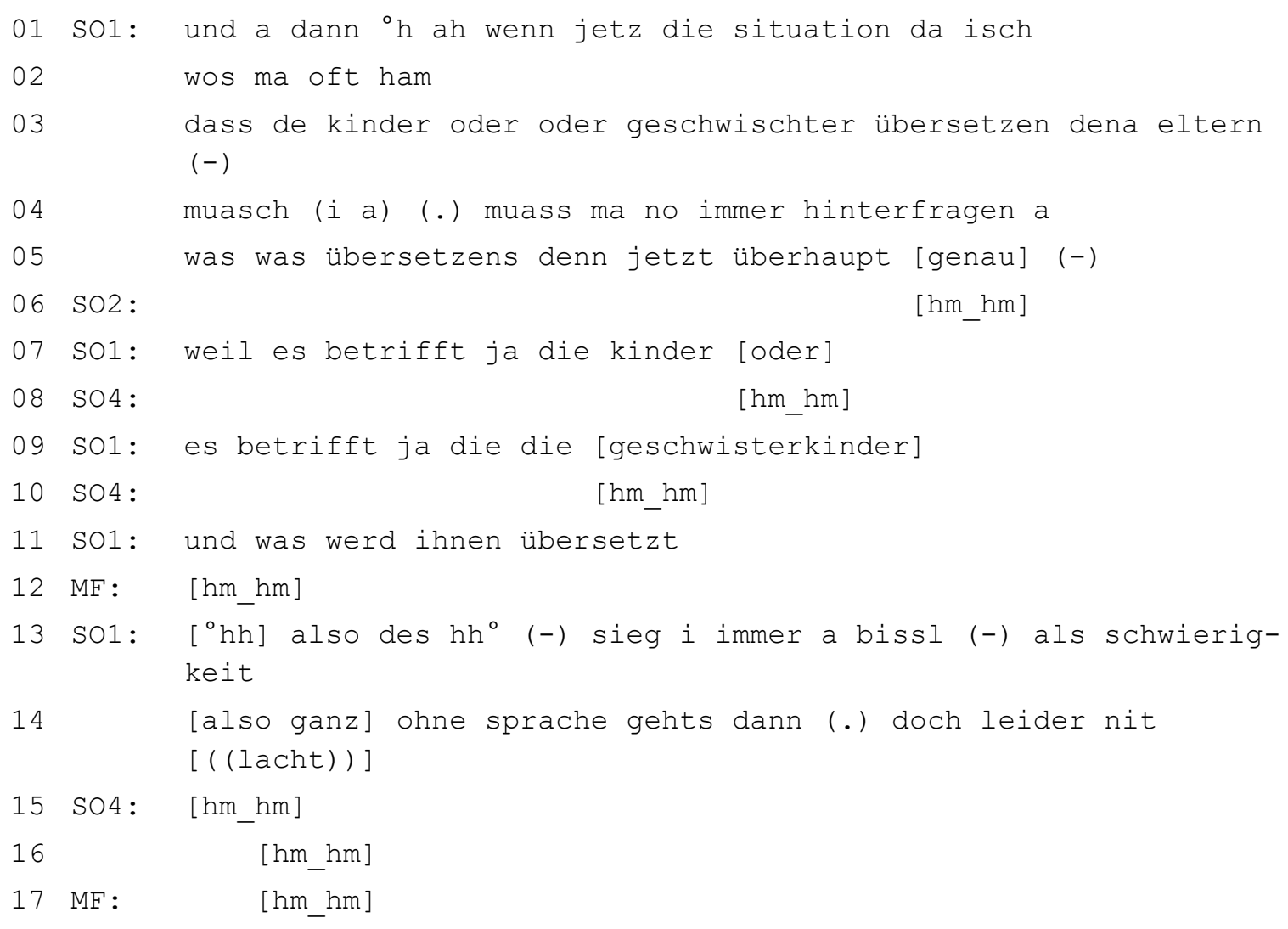

Weitere interaktionale Herausforderungen betreffen die Arbeit mit mehrsprachigen Schülergruppen in Klassensituationen, wo die Gruppengröße das Eingehen auf die sprachlichen Verständnisschwierigkeiten einzelner Personen kaum erlaubt (Bsp. Klasse in GD ab min 01:15:40; siehe auch Kapitel 4.3). SchulsozialarbeiterInnen sind hier wieder auf SchulkollegInnen angewiesen, die quasi in Nebengesprächen simultan- bzw. flüsterdolmetschen (GD ab min 01:16:43).

\section{$6 \quad$ Fazit}

Die Befragungen der SchulsozialarbeiterInnen haben ein Forschungsfeld mit zahlreichen angewandt-gesprächslinguistischen Anknüpfungspunkten beleuchtet. Eine erste inhaltsanalytische Auswertung zeigt vielfältige metasprachlich beschriebene Praktiken, die von den SchulsozialarbeiterInnen in Interaktion mit ihrer soziolinguistisch sehr diversen Zielgruppe eingesetzt werden. Diese reichen von recipient design und Verstehensthematisierungen bis hin zu multimodalen und medialen Praktiken. Nichtsdestoweniger stoßen die SchulsozialarbeiterInnen immer wieder auch auf Sprachbarrieren, die die Arbeit mit bestimmten Personengruppen erschweren bis hin zu verunmöglichen, und die auch Dolmetschdienste nur bedingt abschwächen können. Die SchulsozialarbeiterInnen äußern eine gewisse Ratlosigkeit und sind auf der Suche nach Lösungen (EI ab min 00:42:31), Fortbildungen und Forschungsarbeiten, die ihnen in solchen 
Gesprächssituationen Hilfe böten. Welche Ansätze kann die Linguistik hier bereits liefern? Wo ist weitere Forschung nötig? Gebraucht wird neben theoretischem Wissen über Mehrsprachigkeit, Spracherwerb und Sprachenpolitik so etwas wie die Förderung der Sprachmittlungskompetenz, die die agency und damit die kommunikativen Praktiken der SchulsozialarbeiterInnen erweitert. Dazu kann linguistisches Know-how bzgl. gedolmetschter Gespräche ebenso gehören wie die Ausgabe von Handouts in Leichter Sprache (Bredel/Maßß 2016) bei Elternabenden, die die Tagesordnung und die wichtigsten Informationen umfassen und Eltern mit anderer L1 als Deutsch das Verständnis erleichtern. U. v. m. Aus den Erzählungen der SchulsozialarbeiterInnen geht darüber hinaus hervor, dass linguistisch fundiertes Mehrsprachigkeitswissen in Zusammenhang mit linguistisch fundierter Sprachmittlungs- und Diversitätskompetenz nicht nur für die Schulsozialarbeit, sondern für den gesamten Sozialraum Schule von den LehrerInnen bis zu den SchülerInnen relevant sind. Schule zeigt sich somit als ein Feld, in dem Wissen und Methoden der Angewandten Linguistik gefragt sind.

\section{Literaturverzeichnis}

Agar, Michael (1994): Language Shock. Unterstanding the Culture of Conversation. New York: Morrow.

Arnold, Rolf (2018): „Von der interkulturellen Kompetenz zur Diversitätskompetenz“. In: Busche, Hubertus et al. (eds.): Kultur - Interdisziplinäre Zugänge. Wiesbaden, Springer: 305-329.

Blöbaum, Bernd/Nölleke, Daniel/Scheu, Andreas (2016): „Das Experteninterview in der Kommunikationswissenschaft"،. In: Averbeck-Lietz, Stefanie/Meyen, Michael (eds.): Handbuch nicht standardisierte Methoden in der Kommunikationswissenschaft. Wiesbaden, Springer: 1-13. (= Springer NachschlageWissen).

BMB (Bundesministerium für Bildung) (2016): 9740/AB XXV. GP - Anfragebeantwortung betreffend Chancenindex für Integrationsmaßnahmen. parlament.gv.at/PAKT/VHG/XXV/ AB/AB_09740/imfname_570844.pdf [10.01.2020].

BMBF (Bundesministerium für Bildung und Frauen) (2016): „Unterstützung von Schulaufsicht und Schulen bei der Integration von Flüchtlingskindern und -jugendlichen durch mobile interkulturelle Teams; Kooperation mit dem Österreichischen Zentrum für psychologische Gesundheitsförderung im Schulbereich (ÖZPGS)“. Erlass. schulpsychologie.at/fileadmin/upload/schulpsychologie/erlass_MIT.pdf [18.02.2020].

Bredel, Ursula/Maaß, Christiane (2016): Leichte Sprache. Theoretische Grundlagen Orientierung für die Praxis. Berlin: Dudenverlag.

Brizić, Katharina (2007): Das geheime Leben der Sprachen. Gesprochene und verschwiegene Sprachen und ihr Einfluss auf den Spracherwerb in der Migration. Münster: Waxmann. (= Internationale Hochschulschriften 465).

Busch, Brigitta (2017): Mehrsprachigkeit. 2. Auflage. Wien/Stuttgart: facultas; UTB. (=utbstudi-e-book 3774).

Dannerer, Monika/Franz, Marianne (2019): ,,Weil eigentlich alles international geworden ist‘. Sprachliche Anforderungen an das Hotelpersonal im Spannungsfeld zwischen Gästen, Arbeitsbereichen und Arbeitsmarkt". In: Heimerdinger, Timo/Leonardi, Andrea/Reso, Evelyn (eds.): Hotelpersonal/Il personale alberghiero. Lebens- und Arbeitsalltag im Dienste des 
Tourismus/Vita quotidiana e lavoro nel settore turistico. Innsbruck/Wien/Bozen, Studienverlag: 255-277. (= Tourism \& Museum 7).

Davidson, Brad (2000): „The interpreter as institutional gatekeeper. The sociolinguistic role of interpreters in Spanish-English medical discourse“. Journal of Sociolinguistics 4/3: 379-405.

Deppermann, Arnulf (2013): „Interview als Text vs. Interview als Interaktion. [61 Absätze]“. Forum: Qualitative Sozialforschung / Qualitative Social Research 14/3: Art. 13.

Deppermann, Arnulf/Feilke, Helmuth/Linke, Angelika (2016): „Sprachliche und kommunikative Praktiken. Eine Annäherung aus linguistischer Sicht“. In: Deppermann, Arnulf/Feilke, Helmuth/Linke, Angelika (eds.): Sprachliche und kommunikative Praktiken. Berlin/Bosten, de Gruyter: 1-23. (= Jahrbuch des Instituts für Deutsche Sprache 2015).

Deppermann, Arnulf/Schmitt, Reinhold (2008): „Verstehensdokumentationen. Zur Phänomenologie von Verstehen in der Interaktion“. Deutsche Sprache 36/3: 220-245.

Emanuel, Markus (2017): „Jugendhilfe und Schule. Plädoyer für eine offensiv-emanzipatorische Schulsozialarbeit". In: Hollenstein, Erich et al. (eds.): Handbuch der Schulsozialarbeit. Band 1. Weinheim/Basel, Beltz Juventa: 16-23.

Erll, Astrid/Gymnich, Marion (2011): Interkulturelle Kompetenzen. Erfolgreich kommunizieren zwischen den Kulturen. Stuttgart: Metzler.

Expertenrat für Integration (2019): „Integrationsbericht 2019. Integration in Österreich - Zahlen, Entwicklungen, Schwerpunkte“. bmeia.gv.at/fileadmin/user_upload/Zentrale/Integration/Integrationsbericht_2019/Integrationsbericht_2019.pdf [28.02.2020].

Fleck, Elfie (2013): „Zur Situation von lebensweltlich mehrsprachigen SchülerInnen. Aktuelle Lage und neuere Entwicklungen in der Bildungspolitik“. In: Cillia, Rudolf/Vetter, Eva (eds.): Sprachenpolitik in Österreich. Bestandsaufnahme 2011. Frankfurt am Main, Lang: 9-28. (= Sprache im Kontext 40).

Franz, Marianne (i. V.): „,Was ist denn schon Kultur?‘. Selbst- und Fremdpositionierungen von SchulsozialarbeiterInnen im Kontext kultureller Diversität"“.

Gogolin, Ingrid (2008): Der monolinguale Habitus der multilingualen Schule. 2., unveränd. Aufl. Münster etc.: Waxmann. (=Internationale Hochschulschriften 101).

Gumperz, John (1964): „Linguistic and Social Interaction in Two Communities”. In: American Anthropologist 66: 137-153.

Haddington, Pentti/Mondada, Lorenza/Nevile, Maurice (2013): „Being mobile. Interaction on the move“. In: Haddington, Pentti/Mondada, Lorenza/Nevile, Maurice (eds.): Interaction and mobility. Language and the body in motion. Berlin, de Gruyter: 3-62.

Hansen, Klaus (2011): Kultur und Kulturwissenschaft. Eine Einführung. 4., vollständig überarbeitete Auflage. Tübingen/Basel: Francke. (=UTB Kulturwissenschaft 1846).

Hausendorf, Heiko/Mondada, Lorenza/Schmitt, Reinhold (2012): „Raum als interaktive Ressource. Eine Explikation“. In: Hausendorf, Heiko/Mondada, Lorenza/Schmitt, Reinhold (eds.): Raum als interaktive Ressource. Tübingen, Narr Francke Attempto: 7-36. (= Studien zur deutschen Sprache 62).

Hitzler, Sarah (2012): Aushandlung ohne Dissens? Praktische Dilemmata der Gesprächsführung im Hilfeplangespräch. Wiesbaden: VS Verlag für Sozialwissenschaften. 
Hlavac, Jim (2014): „Participation roles of a language broker and the discourse of brokering. An analysis of English-Macedonian interactions“. Journal of Pragmatics 70: 52-67. doi: 10.1016/j.pragma.2014.06.003.

Imo, Wolfgang (2013): „Ellipsen und Verstehen in der Interaktion“. In: Hennig, Mathilde (ed.): Die Ellipse. Neue Perspektiven auf ein altes Phänomen. Berlin/New York, de Gruyter: 281320. (= Linguistik - Impulse \& Tendenzen 52).

Kadrić, Mira (2016): „Dolmetschen als Dienst am Menschen“. In: Kadrić, Mira/Kaindl, Klaus (eds.): Berufsziel Übersetzen und Dolmetschen. Grundlagen, Ausbildung, Arbeitsfelder. Tübingen, Francke: 103-119. (=UTB 4454).

Kletečka-Pulker, Maria/Parrag, Sabine (2018): „Videodolmetschen als Kommunikationshilfe bei Flüchtlingen“. Pädiatrie \& Pädologie 53/S1: 56-60. doi: 10.1007/s00608-0180588-z.

Locher, Miriam/Watts, Richard (2008): „Relational work and impoliteness. Negotiating norms of linguistic behaviour". In: Bousfield, Derek/Locher, Miriam (eds.): Impoliteness in Language. Studies on its Interplay with Power in Theory and Practice. Berlin, de Gruyter: 77-99.

Lüthje, Corinna (2016): „Die Gruppendiskussion in der Kommunikationswissenschaft“. In: Averbeck-Lietz, Stefanie/Meyen, Michael (eds.): Handbuch nicht standardisierte Methoden in der Kommunikationswissenschaft. Wiesbaden, Springer Fachmedien: 157-173.

Morales, Alejandro/Hanson, William (2005): „Language Brokering. An Integrative Review of the Literature“. In: Hispanic Journal of Behavioral Sciences 27/4: 471-503. doi: $10.1177 / 0739986305281333$.

Neuland, Eva (2013): „Interkulturalität - immer noch eine Herausforderung für Linguistik und Deutsch als Fremdsprache“. In: Zeitschrift des Verbandes Polnischer Germanisten 2/2: 161-177. doi: 10.4467/23534893ZG.14.014.1672.

Pick, Ina (ed.) (2017): Beraten in Interaktion. Eine gesprächslinguistische Typologie des Beratens. Frankfurt am Main etc.: Lang. (=Forum Angewandte Linguistik 60).

Pöllabauer, Sonja (2005): „I don’t understand your English, Miss“. Dolmetschen bei Asylanhörungen. Tübingen: Narr. (= Translations-Wissenschaft 2).

Reimann, Daniel (2017): Interkulturelle Kompetenz. Tübingen: Narr Francke Attempto

Sacks, Harvey/Schegloff, Emanuel/Jefferson, Gail (1974): A Simplest Systematics for the Organization of Turn-Taking for Conversation. In: Language 50/4: 696. doi: 10.2307/412243.

Scheuringer, Hermann (1997): Sprachvarietäten in Österreich. In: Stickel, Gerharhd (ed.): Varietäten des Deutschen. Regional- und Umgangssrpachen. Berlin/New York, de Gruyter: 332-345. (= Jahrbuch des Instituts für Deutsche Sprache 1996)

Schmitt, Reinhold/Deppermann, Arnulf (2009): „,Damit Sie mich verstehen“. Genese, Verfahren und Recipient Design einer narrativen Performance“. In: Buss, Mareike et al. (eds.): Theatralität des sprachlichen Handelns. Eine Metaphorik zwischen Linguistik und Kulturwissenschaften. Paderborn/München, Fink: 79-112.

Schörner, Barbara/Würfl, Christine (2013): „Zum Aufgaben- und Kompetenzprofil von Schulsozialarbeit in Österreich“. soziales_kapital. wissenschaftliches journal österreichischer fachhochschul-studiengänge soziale arbeit 10. soziales-kapital.at/index.php/sozialeskapital/article/viewFile/288/479.pdf [28.02.2020]. 
Schulpsychologie, Bildungsberatung/BMBWF, Bundesministerium für Bildung, Wissenschaft und Forschung: „Schulsozialarbeit. Verbindung von schulischer und außerschulischer Lebenswelt". schulpsychologie.at/schuelerber/schulsozialarbeiterinnen [18.02.2020].

Speck, Karsten (2014): Schulsozialarbeit. Eine Einführung; mit 11 Tabellen; mit Prüfungsfragen und-antworten. 3., überarb. und erw. Aufl. München: Reinhardt. (= UTB 2929).

Spitzmüller, Jürgen (2017): „,Kultur' und ,das Kulturelle‘. Zur Reflexivität eines begehrten Begriffs“. Zeitschrift für Angewandte Linguistik 67/1: 3-23. doi: 10.1515/zfal-2017-0016.

STATcube: Statistische Datenbank von Statistik Austria. statcube.at/statistik.at/ext/statcube/jsf/tableView/tableView.xhtml [16.07.2020].

verbal (2019): „Stellungnahme des Verbands für Angewandte Linguistik Österreich zum Integrationsbericht 2019 des ,Expertenrats für Integration““. Im Namen des Vorstands von verbal erstellt durch Alexandra Wojnesitz und Jürgen Spitzmüller. verbal.at/fileadmin/ user_upload/Stellungnahmen/Verbal_Stellungnahme_Integrationsbericht_2019_final.pdf. [20.07.2020].

Wilde, July de et al. (2018): „Shifting multilingual strategies in a Flemish public healthcare service“. Multilingua 37/4: 377-401. doi: 10.1515/multi-2017-0108.

Zwengel, Almut (2015): „Strategien der Interessenvertretung und der Verständnissicherung. Wenn Kinder Gespräche zwischen eingewanderten Müttern und Lehrpersonen dolmetschen“. In: Hauser, Stefan/Mundwiler, Vera (eds.): Sprachliche Interaktion in schulischen Elterngesprächen. Bern, hep der Bildungsverlag: 125-149. 